\title{
Cough without a cause: Habit cough / Tic cough
}

\author{
Shah $\mathrm{AD}^{1,}$ Apoorva $\mathrm{BVS}^{2 *}$, Jain $\mathrm{A}^{2}$, Shah $\mathrm{KV}^{3}$ \\ 1. Professor and HOD Department of Respiratory Medicine ,Smt S.B.K.S Medical Institute\& Research,Sumandeep Vidyapeeth, Vadodara \\ 2. $2^{\text {nd }}$ year PG Student, Department of Respiratory Medicine, Smt S.B.K.S Medical Institute\& Research,Sumandeep Vidyapeeth, Vadodara \\ 3. Professor, Department of Respiratory Medicine, Smt S.B.K.S Medical Institute\& Research,Sumandeep Vidyapeeth, Vadodara
}

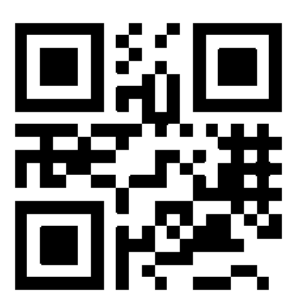

\begin{abstract}
:
Background: Habit cough is a chronic condition commonly seen in children. Previously many case reports have been published in pediatric population but there are very few cases reported in adults. We have reported three different clinical presentations in young adults who presented to our respiratory medicine OPD with a complaint of chronic persistent cough for which they had consulted many physicians and had undergone various investigations and received multiple courses of antihistaminic, antibiotics and oral steroids without any improvement and remained
\end{abstract} undiagnosed. After admission a diagnosis of habit cough was made after ruling out all other underlying medical conditions and was treated with sessions of hypnotherapy and relaxation techniques along with pharmacologic treatment with anxiolytics. After few sessions of hypnotherapy, they were relieved from this ailment. This case series is to bring out the importance of considering habit cough as a diagnosis in young adults without an organic cause. Efforts to make an early diagnosis will reduce morbidity, prevent fixation of symptoms and avoid unnecessary procedures and therapies.

Key words: Habit Cough, Psychogenic cough, Tic cough

\section{*Corresponding Author}

Dr. B V S Apoorva

E-mail id: apoorvabvs@gmail.com

Copyright: This is an open-access article distributed under the terms of the Creative Commons Attribution license which permits unrestricted use, distribution, and reproduction in any medium for non-commercial use (Non Commercial, or CC-BY-NC) provided the original author and source are credited.

\section{INTRODUCTION}

Psychogenic cough also called 'barking cough' or 'honking cough' or 'habit cough 'is defined as a chronic cough (>8 weeks duration) that occurs in the absence of any underlying disease. It is usually loud and harsh, honking or barking in nature, persistent and disruptive to routine activity. ${ }^{1}$ It may become a debilitating condition of long duration that can significantly interfere with work and social relationships. It is mostly seen in children ${ }^{2}$ and usually misdiagnosed as asthma. There are very few reports in adults. Diagnosis of psychogenic cough is clinically challenging ${ }^{3}$ especially in Indian health setup. This case series is an attempt to create awareness to consider the possibility of habit cough as a cause of chronic cough in young adults.

CASE 1 :A 16 year old female presented to our respiratory medicine OPD with complaints of dry cough since 3 months .Cough was sudden in onset, repetitive and honking in nature with each bout lasting for about 1 minute. It worsened while talking and was absent during sleep. No history of past or recent upper respiratory infection, haemoptysis ,chest pain, fever, wheezing, post nasal drip, anorexia or weight loss. She was a non smoker and no history of any substance abuse. Patient had similar complaints 6 months back for which she was admitted elsewhere and took symptomatic treatment with anti-histaminic, steroids and antibiotics but the symptoms persisted. On examination she was moderately built and healthy. Upper and lower respiratory tract examination was normal. Laboratory parameters like complete blood picture, ESR, Differential count, chest $\mathrm{x}$ ray, $\mathrm{X}$ ray PNS,PFT and CT thorax were normal. .Foreign body was suspected and bronchoscopy was done which was also normal. Despite detailed evaluation, no organic cause was found ,hence possibility of diagnosis of habit cough was made and she was referred to psychiatry department of our hospital. After psychiatric evaluation, she was found to have anxiety disorder. She 
was started on anxiolytics. She was also taught few breathing exercises, relaxation techniques and underwent two sessions of hypnotherapy. On follow up after one month, patient was relived of her chronic cough and anxiety. Later the patient was evaluated on weekly visits till the improvement persisted and she stopped coming to the hospital.

CASE 2 : A 13 year old boy presented to our OPD with two months history of continuous coughing that increased since past fifteen days. The cough was sudden in onset and was not associated with fever, chest pain, sore throat, and postnasal drip. On enquiring further, his parents said that the cough is absent while he is sleeping. He had admitted in a hospital elsewhere for similar complaints and evaluated thoroughly but the investigations failed to yield any result. A provisional diagnosis of allergic bronchitis was made and he was put on antihistaminic and steroids. His cough decreased in intensity and he was discharged. However, the cough resurfaced with similar intensity later on. The patient was seen by numerous physicians where again he was thoroughly investigated (sputum test, highresolution computed tomography of lung, a complete allergic profile, and other relevant blood investigations) for his cough without any conclusion. When he came to us, he made a barking type of sound which occurred in recurrent paroxysms at an interval of $30 \mathrm{~s}$ to $1 \mathrm{~min}$, without expectoration and is not associated with respiratory distress. Because of the continuous coughing he complained of chest and abdominal muscle pain .There was no significant family history. Routine physical examination and all laboratory investigations were unyielding .Despite detailed evaluation, no organic cause was found. He was referred to a psychiatrist in our hospital and psychiatric evaluation was also normal. Here the stressor was the patient was worried about his upcoming board exams. He was treated with cognitive behavioral therapy, reassurance and counselling with a psychologist. Psycho-education was also given to parents to be more supportive of the child. Further follow up showed decreased frequency of cough.
CASE 3 :A 25 year-old woman, with complaints of dry cough, episodes of hyperventilation, sadness since two months, decreased sleep since one month.

Cough was abrupt in onset, recurrent, lasted for upto 3-4 minutes with spontaneous reduction in episodes. No history of sneezing, running nose, fever, loss of weight. No history of asthma, tuberculosis, hypertension, diabetes, previous hospitalizations. On enquiring further she had a past history of history of white discharge per vagina since 6 months and was advised for hysterectomy for the same elsewhere. All the Lab investigations, $X$ ray chest, PNS, PFT were normal. As all other medical conditions were ruled out and no cause was found, a diagnosis of habit Cough/Psychogenic cough was suspected in view of recent psychological trigger in the form of hysterectomy. For white discharge per vagina she was referred to $\mathrm{OBG}$ department where she was thoroughly evaluated. USG abdomen and pelvis was normal and she was symptomatic treatment for the same and advised against hysterectomy. She was referred to psychiatry department for further treatment. She was treated with reassurance and behavioral therapy. For her the stressor for the patient was pressure from family to undergo hysterectomy which further lead to manifestation of symptoms of habit cough. Once she was reassured that she doesn't need the surgery, her symptoms subsided. On follow up, cough and episodes of hyperventilation subsided and she was symptomatically better.

DISCUSSION Irwin et al suggests that the prevalence of psychogenic cough is a matter of debate, it is diagnosed in around 3\%-10\% in children presenting with chronic cough of unknown origin. ${ }^{4}$ However, a Chinese systematic review and meta-analysis conducted by Wei et al. ${ }^{5}$ in 2016 reported the overall prevalence of psychogenic cough as $3.02 \%$ (95\% confidence interval: $2.53 \%-$ $3.60 \%$ ). The diagnosis of habit cough is a diagnosis of exclusion that can be made only after an extensive evaluation has been performed to rule out other disorders. This extensive investigation is important to assure both the physician and the patient that no 
major disease is being missed $^{6}$

Table 1: Proposed diagnostic criteria for habit cough in adults by Ramanuja et $\mathrm{al}^{7}$

- Cough is chronic (>3-week duration)

- Cough not explained by an organic cause after a thor diagnostic evaluation

- Cough not responsive to combined therapy for postna syndrome, asthma, or gastroesophageal reflux diseas

- Cough is not intentionally produced or feigned (maling factitious disorders)

- At least 3 or more of the following clinical characterist present:

Female sex

Single or widowed

Cough does not awaken patient from sleep

Underlying psychopathologic condition is present (if depression, anxiety, or somatoform disorder)

Cough disrupts normal social activities and worsens contacts (eg, family, health care professionals, tel conversations)

Secondary gain is associated with the cough

Cough precipitated by emotional distress

"La belle indifference" is displayed despite severity of th

Habit cough is more common in females which was seen even in our study (two females, one male).All three patients were young adults with the age group of less than 30 years which is in accordance with literature. Few case reports have shown that habit cough occurs in older adults as in study by Himanshu sharma et al. ${ }^{8}$ In our study it was found that two out of three patients had barking/honking nature of cough and cough that subsides during sleep. Similarly two of them had psychological issues/triggers.

It is possible that the relationship between psychological issues and chronic cough is different between individuals. A model proposing the complex relationship between psychological issues and chronic cough is presented in Figure 1 and is explained in study by Vertigan $\mathrm{AE}$ et al .This figure suggests that co-existing psychological issues in chronic cough may a causal factor in the pathogenesis of cough, a side effect of chronic cough, or be unrelated. ${ }^{9}$

All three patients responded to cognitive psychotherapy

(non-pharmacological intervention) and reassurance. One patient required anxiolytics in the form of clonazepam in view of associated anxiety disorder along with habit cough.
A

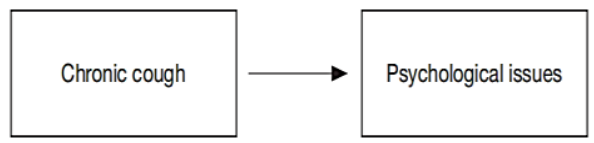

B

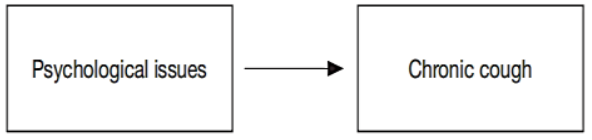

C

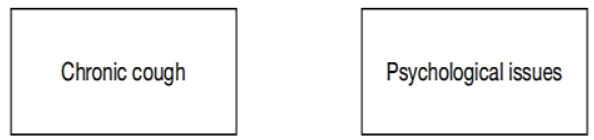

Figure 1 Three different relationships between psychological issues and chronic cough. (A) Cough is a result of psychomorbidity; (B) psychomorbidity is the result of cough; (C) cough and psychomorbidity co-exist but have no causal relationship.

\section{Table 2: Non Pharmacological interventions.}

\begin{tabular}{|c|}
\hline Hypnothery \\
\hline $\begin{array}{l}\text { Suggestion Therapy with /without a } \\
\text { distractor }\end{array}$ \\
\hline $\begin{array}{l}\text { Mix of multiple interventions, including } \\
\text { reassurance, counselling, referral to } \\
\text { psychologist, } \\
\text { medications }\end{array}$ \\
\hline $\begin{array}{l}\text { Voice therapy techniques } \\
\text { psychotherapy }\end{array}$ \\
\hline Vocal fold injection \\
\hline
\end{tabular}

In this case series no etiology was found for chronic cough despite through medical evaluation and hence they were labelled with the diagnosis of habit cough. It suggests that habit cough is as treatable in adults as in pediatric patients. It is mandatory that any patient with a chronic cough receive a thorough medical workup to eliminate the possibility of medical condition and after that only be labelled with habit/psychogenic cough. It is also important that the clinician makes it clear to such patients and their care-taker about the nature of the illness and that psychotherapy, behavior therapy and other relaxation techniques are specific treatments. It is necessary to recognize this condition early in its course so that appropriate treatment can be given so as to decrease the burden of illness and improve the quality of life. ${ }^{8}$ 


\section{CONCLUSION}

It is important to consider habit cough in the differential diagnosis of chronic cough because an early diagnosis will reduce morbidity, prevent fixation of symptoms and avoid unnecessary procedures and therapies. In young people with more severe symptoms, the provision of coping strategies and increasing the subjective sense of control is an intervention in itself and will improve the likelihood of a good outcome. In extreme cases, the role of rehabilitation programmes involving negotiation with schools and community organizations may prove useful in remediation of the cough and normalization of social and peer supports.

\section{REFERENCES}

1. Weinberger M, Abuhasan M. Pseudoasthma: when cough, wheezing and dyspnoea are not asthma. Pediatrics 2007; 120:855-64.

2. McGarvey LP, Warke TJ, McNiff C, Heaney LG. Psychogenic cough in a schoolboy: evaluation using an ambulatory cough recorder. Pediatr Pulmonol 2003; 36:73-5

3. Jakati PK, Naskar S, Khanna A. "The Barking Girl": A Case Report of Psychogenic Cough in a Child with a Review of Literature. Indian J Psychol Med. 2017;39(4):542-545.

4. Irwin RS, Glomb WB, Chang AB. Habit cough, tic cough, and psychogenic cough in adult and pediatric populations: ACCP evidence-based clinical practice guidelines. Chest 2006;129 1 Suppl: 174S-9S

5. Wei W, Zhangtong Y, Li H, Hou J, Lv $\mathrm{H}$, Li C. Detection rate of psychogenic cough in patients with chronic cough Indian Journal of Psychological Medicine Volume 39 Issue 4 JulyAugust 2017545 in Chinese hospital: A meta analysis. IntJ Clin Exp Med 2016;9:504-14.

6. Ramanuja S, Kelkar P. Habit Cough. Ann Allergy Asthma Immunol 2009;102:91-5.

7. Mastrovich JD, Greenberger PA. Psychogenic cough in adults: a report of two cases and review of the literature. Allergy Asthma Proc. 2002; 23:27-33.
8. Sharma H, Thaker S. Chronic psychogenic cough in an adult patient of schizophrenia. Int $\mathbf{J}$ Health Sci Res. 2017; 7(9):307-310

9. Vertigan AE. Somatic cough syndrome or psychogenic cough-what is the difference? J Thorac Dis 2017;9(3):831838. doi: 10.21037/jtd.2017.03.119. 